\title{
Parabolic conjugacy in general linear groups
}

\author{
Simon M. Goodwin • Gerhard Röhrle
}

Received: 27 November 2006 / Accepted: 6 April 2007 /

Published online: 15 June 2007

(C) Springer Science+Business Media, LLC 2007

\begin{abstract}
Let $q$ be a power of a prime and $n$ a positive integer. Let $P(q)$ be a parabolic subgroup of the finite general linear group $\mathrm{GL}_{n}(q)$. We show that the number of $P(q)$-conjugacy classes in $\mathrm{GL}_{n}(q)$ is, as a function of $q$, a polynomial in $q$ with integer coefficients. This answers a question of Alperin in (Commun. Algebra 34(3): 889-891, 2006)
\end{abstract}

Keywords General linear group · Parabolic subgroups · Conjugacy classes

\section{Introduction}

Let $\mathrm{GL}_{n}(q)$ be the general linear group of nonsingular $n \times n$ matrices over the finite field $\mathbb{F}_{q}$, and let $\mathrm{U}_{n}(q)$ be the subgroup of $\mathrm{GL}_{n}(q)$ consisting of upper unitriangular matrices. A longstanding conjecture states that the number of conjugacy classes of $\mathrm{U}_{n}(q)$ is, as a function of $q$, a polynomial in $q$ with integer coefficients. This conjecture has been attributed to Higman cf. [7] and verified by computer for $n \leq 13$ by Vera-López and Arregi [15]. There has been further interest in this conjecture from Robinson [12] and Thompson [14].

In [1], Alperin showed that a related result is "easily established", namely, that the number of $\mathrm{U}_{n}(q)$-conjugacy classes in all of $\mathrm{GL}_{n}(q)$ is a polynomial in $q$ with integer coefficients. This theorem can be viewed as evidence in support of Higman's conjecture. Alperin also considers the possibility of a proof of Higman's conjecture

\footnotetext{
S.M. Goodwin

School of Mathematics, University of Birmingham, Birmingham, B15 2TT, UK

e-mail: goodwin@maths.bham.ac.uk

G. Röhrle ( $₫)$

Fakultät für Mathematik, Ruhr-Universität Bochum, Universitätsstrasse 150, 44780 Bochum,

Germany

e-mail: gerhard.roehrle@rub.de
} 
by descent from the theorem proved in [1], though he says that this seems very unlikely.

In addition, Alperin showed in [1] that the number of $\mathrm{B}_{n}(q)$-conjugacy classes in $\mathrm{GL}_{n}(q)$ is a polynomial in $q$, where $\mathrm{B}_{n}(q)$ is the subgroup of upper triangular matrices in $\mathrm{GL}_{n}(q)$.

Let $d=\left(d_{1}, \ldots, d_{t}\right) \in \mathbb{Z}_{\geq 1}^{t}$ satisfy $d_{i}<d_{i+1}$ and $d_{t}=n$; we call such $d$ an $n$ dimension vector. Let $\mathrm{P}_{n, d}(\bar{q})$ be the parabolic subgroup of $\mathrm{GL}_{n}(q)$ that stabilizes the standard flag $\{0\} \subseteq \mathbb{F}_{q}^{d_{1}} \subseteq \mathbb{F}_{q}^{d_{2}} \subseteq \cdots \subseteq \mathbb{F}_{q}^{d_{t}}=\mathbb{F}_{q}^{n}$, and let $\mathrm{U}_{n, d}(q)$ be the unipotent radical of $\mathrm{P}_{n, d}(q)$. In [1], Alperin asks whether the number of $\mathrm{U}_{n, d}(q)$-conjugacy classes in $\operatorname{GL}_{n}(q)$ is a polynomial in $q$; and likewise for the number of $\mathrm{P}_{n, d}(q)$ conjugacy classes in $\mathrm{GL}_{n}(q)$. In [5, Theorem 4.5], the authors showed that this question for $\mathrm{U}_{n, d}(q)$ has an affirmative answer. In this paper, we prove the following theorem, which affirmatively answers Alperin's question for $\mathrm{P}_{n, d}(q)$.

Theorem 1.1 The number of $\mathrm{P}_{n, d}(q)$-conjugacy classes in $\mathrm{GL}_{n}(q)$ is, as a function of $q$ for fixed $d$, a polynomial in $q$ with integer coefficients.

The special case of Theorem 1.1 where $\mathrm{P}_{n, d}(q)=\mathrm{GL}_{n}(q)$ is of course well known.

In order to state a proposition related to Theorem 1.1, we need to recall some standard terminology. We let $K$ be the algebraic closure of $\mathbb{F}_{q}$ and view $\operatorname{GL}_{n}(q)$ as a subgroup of $\mathrm{GL}_{n}(K)$ in the natural way. Recall that two parabolic subgroups of $\mathrm{GL}_{n}(K)$ are said to be associated if they have Levi subgroups that are conjugate in $\mathrm{GL}_{n}(K)$. We write $\mathrm{P}_{n, d}(K)$ for the parabolic subgroup of $\mathrm{GL}_{n}(K)$ such that $\mathrm{P}_{n, d}(K) \cap \mathrm{GL}_{n}(q)=\mathrm{P}_{n, d}(q)$. Let $d=\left(d_{1}, \ldots, d_{t}\right)$ and $d^{\prime}=\left(d_{1}^{\prime}, \ldots, d_{t^{\prime}}^{\prime}\right)$ be $n$ dimension vectors. We recall that $\mathrm{P}_{n, d}(K)$ and $\mathrm{P}_{n, d^{\prime}}(K)$ are associated if and only if $t=t^{\prime}$ and there exists $\sigma \in \operatorname{Sym}(t)$ such that $d_{i}-d_{i-1}=d_{\sigma i}^{\prime}-d_{\sigma i-1}^{\prime}$ for all $i=1, \ldots, t$; by convention, we set $d_{0}=d_{0}^{\prime}=0$.

By $[5,(4.15)]$ we have the following proposition. We indicate how it is proved in the outline of the proof of Theorem 1.1 given below.

Proposition 1.2 Let $\mathrm{P}_{n, d}(K)$ and $\mathrm{P}_{n, d^{\prime}}(K)$ be associated parabolic subgroups of $\mathrm{GL}_{n}(K)$. Then the number of $\mathrm{P}_{n, d}(q)$-conjugacy classes in $\mathrm{GL}_{n}(q)$ is equal to the number of $\mathrm{P}_{n, d^{\prime}}(q)$-conjugacy classes in $\mathrm{GL}_{n}(q)$.

We note that the proof of the observation in Proposition 1.2 does not yield a bijection between the two sets of orbits. It would be interesting to know if a bijection can be defined in a natural way.

Below we give an outline of our proof of Theorem 1.1. Before doing this, we simplify our notation. We write $G=\mathrm{GL}_{n}(q), B=\mathrm{B}_{n}(q)$, and, for $d$ as above, $P=$ $\mathrm{P}_{n, d}(q)$. For a subgroup $H$ of $G$, we write $k(H, G)$ for the number of $H$-conjugacy classes in $G$. Although this notation does not show a dependence on $q$, we want to allow $q$ to vary and, for $G, B, P$, to define groups for each $q$; so, for example, it makes sense to say that $k(P, G)$ is a polynomial in $q$. We write $\mathbf{G}=\mathrm{GL}_{n}(K)$ and $\mathbf{P}$ for the parabolic subgroup of $\mathbf{G}$ corresponding to $P$.

For $x \in G$, we define $f_{P}^{G}(x)$ to be the number of conjugates of $P$ containing $x$, i.e., $f_{P}^{G}(x)=\left|\left\{{ }^{y} P \mid y \in G, x \in{ }^{y} P\right\}\right|$. A counting argument as in [1] (see also [5, 
$\S 4.1])$, along with the fact that $P=N_{G}(P)$, yields

$$
k(P, G)=\sum_{x \in \mathcal{R}} f_{P}^{G}(x),
$$

where $\mathcal{R}=\mathcal{R}(P, G)$ is a set of representatives of the conjugacy classes of $G$ that intersect $P$. We note that if the conjugacy class of $x \in G$ misses $P$, then $f_{P}^{G}(x)=0$. Therefore, it does no harm in (1.1) to sum over a set of representatives $\mathcal{R}=\mathcal{R}(G)$ of all conjugacy classes of $G$.

From the proof of [5, Lemma 3.2] one can observe that, for $x \in G, f_{P}^{G}(x)$ only depends on $P$ up to the association class of $\mathbf{P}$, i.e., if $\mathbf{P}$ and $\mathbf{Q}$ are associated parabolic subgroups of $\mathbf{G}$, then $f_{P}^{G}(x)=f_{Q}^{G}(x)$ for all $x \in G$. This is a consequence of the fact that the Harish-Chandra induction functor $R_{L}^{G}$ is independent of the choise of a parabolic subgroup which contains $L$ as a Levi subgroup. This observation is used to deduce [5, (4.15)] and thus Proposition 1.2.

In [1], Alperin shows that $k(B, G)$ is a polynomial in $q$, using formula (1.1) for the case $P=B$. The proof of this depends on partitioning the set $\mathcal{R}(B, G)$ into a finite union $\mathcal{R}(B, G)=\mathcal{R}_{1} \cup \cdots \cup \mathcal{R}_{r}$ independent of $q$ (though some $\mathcal{R}_{i}$ may be empty for small $q$ ) such that $f_{B}^{G}(x)=f_{B}^{G}(y)$ if $x, y \in \mathcal{R}_{i}$; and $\left|\mathcal{R}_{i}\right|$ is a polynomial in $q$. An inductive counting argument is used to show that $f_{B}^{G}\left(x_{i}\right)$ is given by a polynomial in $q$ for $x_{i} \in \mathcal{R}_{i}$.

In this paper, we give an analogous decomposition $\mathcal{R}(G)=\mathcal{R}_{1} \cup \cdots \cup \mathcal{R}_{r}$; this partition is based on Jordan normal forms. Again, this decomposition does not depend on $q$ (though some $\mathcal{R}_{i}$ may be empty for small $q$ ), and we show that $\left|\mathcal{R}_{i}\right|$ is a polynomial in $q$. Let $x \in \mathcal{R}_{i}$, for some $i$, with Jordan decomposition $x=s u$, and let $H=C_{G}(s)$. We show that $f_{P}^{G}(x)$ can be expressed as a sum of terms of the form $f_{Q}^{H}(u)$, where $Q$ is a parabolic subgroup of $H$ of the form ${ }^{y} P \cap H$ for some $y \in G$. If $x^{\prime}=s^{\prime} u^{\prime} \in \mathcal{R}_{i}$, then we have $u^{\prime}=u$ and so we have $f_{P}^{G}\left(x^{\prime}\right)=f_{P}^{G}(x)$. We can appeal to [5, Theorem 3.10] to deduce that each $f_{Q}^{H}(u)$ is a polynomial in $q$ and, therefore, that $f_{P}^{G}(x)$ is a polynomial in $q$. The key point in the proof that $f_{Q}^{H}(u)$ is a polynomial in $q$ is to show that it can be expressed in terms of Green functions; in the present setting, the results in [6] show that these Green functions are polynomials in $q$. We then have

$$
k(P, G)=\sum_{i=1}^{r}\left|\mathcal{R}_{i}\right| f_{P}^{G}\left(x_{i}\right),
$$

where $x_{i} \in \mathcal{R}_{i}$. Each summand on the right-hand side of (1.2) is a polynomial in $q$. Hence, $k(P, G)$ is a polynomial in $q$.

We are left to show that, as a polynomial in $q, k(P, G)$ has integer coefficients. This is nontrivial: although the coefficients of the polynomial $f_{P}^{G}(x)$ are integers (this follows from the results in [5, §4]), the coefficients of the polynomials $\left|\mathcal{R}_{i}\right|$ are not integers in general. In order to show that $k(P, G) \in \mathbb{Z}[q]$, we argue that the $P$ conjugacy classes in $G$ can be parameterized by the $\mathbb{F}_{q}$-rational points of a family of varieties defined over $\mathbb{F}_{q}$. Then we apply some standard arguments.

Let $U$ be the unipotent radical of $P$, and let $u \in G$ be unipotent. Using the theory of Green functions, it is proved in [5] that $f_{U}^{G}(u)$ is a polynomial of $q$; also in the 
appendix of loc. cit., an elementary counting argument is used to give an alternative proof of this. It is possible to give an elementary proof that $f_{P}^{G}(u)$ is a polynomial in $q$ for unipotent $u$; this proof is similar to that in the appendix to [5] and is rather technical, so we choose not to include it here. Given such a proof, one can avoid appealing to the theory of Green functions in the proof of Theorem 1.1. For this one needs to observe that, for semisimple $s \in G$, the centralizer $H=C_{G}(s)$ is isomorphic to a direct product of groups of the form $\operatorname{GL}_{m}\left(q^{l}\right)$, where $m, l \in \mathbb{Z}_{\geq 1}$. Then, for arbitrary $x \in G$ with Jordan decomposition $x=s u$, one can deduce that $f_{P}^{G}(x)$ is a polynomial in $q$ using the expression for $f_{P}^{G}(x)$ as a sum of terms of the form $f_{Q}^{H}(u)$.

In analogy to a comment made at the end of the appendix to [5], it is not possible to deduce Proposition 1.2 from an elementary proof of Theorem 1.1 as described above.

One can consider the more general situation where the general linear group $\mathrm{GL}_{n}(q)$ is replaced by an arbitrary finite group of Lie type $G$, and $P$ is a parabolic subgroup of $G$ with unipotent radical $U$. The precise formulation of the analogous questions regarding $k(U, G)$ and $k(P, G)$ being polynomials in $q$ with integer coefficients is rather technical, so we do not give it here; this formulation requires an axiomatic setup as in [5, §2.2]. However, we note that [5, Theorem 4.5] says that $k(U, G)$ is a polynomial in $q$ if $p$ is $\operatorname{good}$ for $\mathbf{G}$ and $\mathbf{G}$ has connected centre, where $\mathbf{G}$ is the connected reductive algebraic group defined over $\mathbb{F}_{q}$ so that $G$ is the group of $\mathbb{F}_{q}$-rational points of $\mathbf{G}$. In the case $\mathbf{G}$ has disconnected centre, $k(U, G)$ is only given by polynomials up to congruences on $q$. That is, in the language of G. Higman [8], $k(U, G)$ is PORC (Polynomial On Residue Classes); this is discussed before [5, Example 4.10]. The question about $k(P, G)$ is more difficult in general. We believe that one should be able to generalize the arguments in this paper to show that $k(P, G)$ is PORC in general. As is mentioned in [5, Remark 4.12], the centre of a pseudo-Levi subgroup of $\mathbf{G}$ need not be connected even if the centre of $\mathbf{G}$ is connected; therefore, in general, one can only hope to prove that $f_{P}^{G}(x)$ is PORC.

As a general reference for algebraic groups defined over finite fields, we refer the reader to the book by Digne and Michel [2].

\section{Notation}

We establish the notation to be used throughout this note. We continue to use the convention that the objects we define depend on the prime power $q$, but this dependence is suppressed in our notation.

We write $\mathbb{F}_{q}$ for the finite field of $q$ elements. We denote the algebraic closure of $\mathbb{F}_{q}$ by $K$ and we consider all the finite fields $\mathbb{F}_{q^{m}}$ (for $m \in \mathbb{Z}_{\geq 1}$ ) as subfields of $K$. The set of nonzero elements of $K$ is denoted by $K^{\times}$; likewise $\mathbb{F}_{q}^{\times}$denotes the set of nonzero elements of $\mathbb{F}_{q}$. For $a \in K^{\times}$, the degree of a over $q$, denoted $\operatorname{deg}(a)=\operatorname{deg}_{q}(a)$, is the minimal value of $m$ such that $a \in \mathbb{F}_{q^{m}}$. For $m \in \mathbb{Z}_{\geq 2}$, we define $\mathbb{F}_{q^{m}}^{\sharp}$ by

$$
\mathbb{F}_{q^{m}}^{\sharp}=\mathbb{F}_{q^{m}} \backslash \bigcup_{j \mid m} \mathbb{F}_{q^{j}}=\{a \in K \mid \operatorname{deg}(a)=m\} ;
$$

we define $\mathbb{F}_{q}^{\sharp}=\mathbb{F}_{q}^{\times}$. 
We write $F$ for the Frobenius morphism on $K$ corresponding to $q$, i.e., $F(a)=a^{q}$ for all $a \in K$. We let $K^{\times} / F$ denote the set of $F$-orbits in $K^{\times}$; this set is in bijection with the set of all monic irreducible polynomials in $\mathbb{F}_{q}[X] \backslash\{X\}$. Given $a \in K$, we write $\bar{a}$ for the $F$-orbit of $a$ in $K$. Note that the degree function is constant on $F$-orbits in $K^{\times}$, so that, for given $\bar{a} \in K^{\times} / F$, the $\operatorname{degree} \operatorname{deg}(a)$ is well defined. Also, we sometimes consider a sum or product over $K^{\times} / F$ where the summands or factors are indexed by representatives of the $F$-classes in $K^{\times}$; in such situations, each summand or factor only depends on the corresponding element in $K^{\times} / F$.

Given a map $\gamma: K^{\times} / F \rightarrow S$, where $S$ is some set, we write $\gamma_{0}: K^{\times} \rightarrow S$ for the map defined by $\gamma_{0}(a)=\gamma(\bar{a})$. For $m \in \mathbb{Z}_{\geq 1}$, we write $\mathbb{F}_{q^{m}}^{\sharp} / F$ for the set of $F$-orbits in $\mathbb{F}_{q^{m}}^{\sharp}$ and define

$$
\phi(m)=\left|\mathbb{F}_{q^{m}}^{\sharp} / F\right| .
$$

We observe that

$$
\phi(m)=\frac{1}{m} \sum_{j \mid m} \mu(j) q^{m / j},
$$

where $\mu$ is the classical Möbius function, see, for example, [9, §1.13]; in particular, $\phi(m)$ is a polynomial in $q$.

By a partition we mean a sequence of the form $\lambda=\left(\lambda_{1}^{c_{1}}, \ldots, \lambda_{l}^{c_{l}}\right)$, where $\lambda_{i}, c_{i} \in$ $\mathbb{Z}_{\geq 1}$ and $\lambda_{i}>\lambda_{i+1}$; we allow $\lambda$ to be the empty partition, i.e., $l=0, \lambda=()$. Given a partition $\lambda$, we let $|\lambda|=\sum_{i=1}^{l} c_{i} \lambda_{i}$. We write $\mathbb{P}$ for the set of all partitions.

We fix a linear order $\prec$ on $\mathbb{P}$ by setting $\lambda \prec \lambda^{\prime}$ if $|\lambda|<\left|\lambda^{\prime}\right|$ and then ordering the partitions $\lambda$ for fixed $|\lambda|$ lexicographically. By a multi-partition we mean a sequence of the form $\mu=\left(\mu_{1}^{b_{1}}, \ldots, \mu_{m}^{b_{m}}\right)$, where $\mu_{i} \in \mathbb{P}, b_{i} \in \mathbb{Z}_{\geq 1}$, and $\mu_{i} \succ \mu_{i+1}$; we allow $\mu$ to be the empty multi-partition. Given a multi-partition $\mu=\left(\mu_{1}^{b_{1}}, \ldots, \mu_{m}^{b_{m}}\right)$, we let $|\mu|=\sum_{i=1}^{m} b_{i}\left|\mu_{i}\right|$. We write $\mathbb{M P}$ for the set of all multi-partitions.

The polynomial defined below is required to simplify the notation in Sect. 3. For a sequence $b=\left(b_{1}, \ldots, b_{m}\right) \in \mathbb{Z}_{\geq 1}^{m}$, we define the following polynomial in the indeterminate $z$ :

$$
\Delta(b, z)=\left(\begin{array}{c}
z \\
b_{1}
\end{array}\right)\left(\begin{array}{c}
z-b_{1} \\
b_{2}
\end{array}\right)\left(\begin{array}{c}
z-b_{1}-b_{2} \\
b_{3}
\end{array}\right) \cdots\left(\begin{array}{c}
z-b_{1}-\cdots-b_{m-1} \\
b_{m}
\end{array}\right),
$$

where $\left(\begin{array}{l}z \\ c\end{array}\right)=\frac{z(z-1) \cdots(z-c+1)}{c !}$ for $c \in \mathbb{Z}_{\geq 1}$. We allow $\Delta$ to be defined for different values of $m$. We note that the coefficients of $\Delta(b, z)$ are in general not integers.

Let $n$ be a positive integer. We write $G=\mathrm{GL}_{n}(q)$ and regard it as a subgroup of $\mathbf{G}=\mathrm{GL}_{n}(K)$. We write $F$ for the standard Frobenius morphism on $\mathbf{G}$ and its natural module $K^{n}$. Therefore, $G=\mathbf{G}^{F}$ is the group of fixed points of $F$ in $\mathbf{G}$, and $\mathbb{F}_{q}^{n}=\left(K^{n}\right)^{F}$.

For $g, x \in G$, we write ${ }^{g} x=g x g^{-1}$; similarly, for a subgroup $H$ of $G$, we write ${ }^{g} H=g \mathrm{Hg}^{-1}$. We write $C_{G}(x)=\left\{\left.g \in G\right|^{g} x=x\right\}$ for the centralizer of $x$ in $G$; the centralizer of $x$ in $\mathbf{G}$ is denoted by $C_{\mathbf{G}}(x)$.

Let $m \in \mathbb{Z}_{\geq 1}$ and $a \in K$. Then the $m \times m$ Jordan matrix $J(a, m)$ is defined as usual. Given a partition $\lambda=\left(\lambda_{1}^{c_{1}}, \ldots, \lambda_{l}^{c_{l}}\right)$, the matrix $J(a, \lambda)$ is defined as a direct 
sum of Jordan matrices:

$$
J(a, \lambda)=\bigoplus_{i=1}^{l} c_{i} J\left(a, \lambda_{i}\right)
$$

Finally, for $\bar{a} \in K^{\times} / F$ and $\lambda \in \mathbb{P}$, we define the matrix

$$
J(\bar{a}, \lambda)=\bigoplus_{i=0}^{\operatorname{deg}(a)-1} J\left(F^{i}(a), \lambda\right) .
$$

By choosing a basis of the form $\mathbb{B}_{0} \cup \mathbb{B}_{1} \cup \cdots \cup \mathbb{B}_{\operatorname{deg}(a)-1}$ for $K^{n}$ (where $n=$ $\operatorname{deg}(a)|\lambda|)$ with $\left|\mathbb{B}_{i}\right|=|\lambda|$ and $F^{i}\left(\mathbb{B}_{0}\right)=\mathbb{B}_{i}$, the matrix $J(\bar{a}, \lambda)$ is fixed by $F$ and so lies in $G$.

\section{The conjugacy classes of $\mathrm{GL}_{n}(q)$}

In this section, we recall the parametrization of the conjugacy classes of $G=\operatorname{GL}_{n}(q)$, see, for example, [10, Ch. IV §2]. We use this parametrization to define the partition of the set of conjugacy classes of $G$ mentioned in the introduction.

The conjugacy classes of $G$ are given by Jordan normal forms, and these are parameterized by maps

$$
\gamma: K^{\times} / F \rightarrow \mathbb{P}
$$

such that $\gamma(\bar{a})$ is the empty partition for all but finitely many $\bar{a} \in K^{\times} / F$ and

$$
\sum_{a \in K^{\times}}\left|\gamma_{0}(a)\right|=\sum_{\bar{a} \in K^{\times} / F} \operatorname{deg}(a)|\gamma(\bar{a})|=n .
$$

We write $\Gamma$ for the set of all such maps $\gamma$. Given $\gamma \in \Gamma$, we can define a linear map $x(\gamma) \in G$ as follows: We decompose $K^{n}$ as

$$
K^{n}=\bigoplus_{a \in K^{\times}} V_{a},
$$

where $\operatorname{dim} V_{a}=\left|\gamma_{0}(a)\right|=|\gamma(\bar{a})|$ and $F\left(V_{a}\right)=V_{F(a)}$ for all $a \in K^{\times}$. For $\bar{a} \in K^{\times} / F$, we write $V_{\bar{a}}=\bigoplus_{i=0}^{\operatorname{deg}(a)-1} V_{F^{i}(a)}$. With respect to an (ordered) basis, denoted $\mathbb{B}(\gamma)_{\bar{a}}$, of $V_{\bar{a}}$, the action of $x(\gamma)$ on $V_{\bar{a}}$ is given by the matrix $J(\bar{a}, \gamma(\bar{a}))$. The set $\{x(\gamma) \mid$ $\gamma \in \Gamma\}$ gives a complete set of representatives of the conjugacy classes of $G$.

For $a \in K^{\times}$, we define $\mathbb{B}(\gamma)_{a}=\mathbb{B}(\gamma)_{\bar{a}} \cap V_{a}$. We write $\mathbb{B}(\gamma)$ for the basis of $K^{n}$ given by $\mathbb{B}(\gamma)=\bigcup_{a \in K^{\times}} \mathbb{B}(\gamma)_{a}$.

Let $\gamma \in \Gamma$. We write the Jordan decomposition of $x(\gamma)$ as $x(\gamma)=s(\gamma) u(\gamma)$. It is straightforward to describe the action of $s(\gamma)$ and $u(\gamma)$ on each $V_{a}$ for $a \in K^{\times}$.

The semisimple part $s(\gamma)$ acts on $V_{a}$ as multiplication by $a$. Therefore, we see that the centralizer of $s(\gamma)$ in $\mathbf{G}$ is

$$
C_{\mathbf{G}}(s(\gamma))=\prod_{a \in K^{\times}} \mathrm{GL}\left(V_{a}\right) \cong \prod_{\bar{a} \in K^{\times} / F} \operatorname{GL}_{|\gamma(\bar{a})|}(K)^{\operatorname{deg}(a)} .
$$


In order to describe the centralizer of $s(\gamma)$ in $G$, we note that $V_{a}$ is defined over $\mathbb{F}_{q^{\operatorname{deg}(a)}}$, and $V_{a}^{F^{\operatorname{deg}(a)}} \cong \mathbb{F}_{q^{\operatorname{deg}(a)}}^{\left|\gamma_{0}(a)\right|}$. Note that, for $a, b \in K^{\times}$in the same $F$-orbit, we have $V_{a}^{F^{\operatorname{deg}(a)}} \cong V_{b}^{F^{\operatorname{deg}(b)}}$. Therefore, as $F\left(V_{a}\right)=V_{F(a)}$, we see that the centralizer of $s(\gamma)$ in $G$ is

$$
C_{G}(s(\gamma)) \cong \prod_{\bar{a} \in K^{\times} / F} \operatorname{GL}\left(V_{a}^{F^{\operatorname{deg}(a)}}\right) \cong \prod_{\bar{a} \in K^{\times} / F} \operatorname{GL}_{|\gamma(\bar{a})|}\left(q^{\operatorname{deg}(a)}\right) .
$$

We write $H(\gamma)=C_{G}(s(\gamma))$.

The action of the unipotent part $u(\gamma)$ on $V_{a}$ is given by the Jordan matrix $J\left(1, \gamma_{0}(a)\right)$ with respect to the basis $\mathbb{B}(\gamma)_{a}$ of $V_{a}$.

Next we define an equivalence relation on $\Gamma$ that gives rise to the desired partition of the conjugacy classes of $G$. For $\gamma, \delta \in \Gamma$, we write $\gamma \sim \delta$ if there is a degreepreserving bijection $\Upsilon: K^{\times} / F \rightarrow K^{\times} / F$ such that $\gamma=\delta \Upsilon$. This defines an equivalence relation on $\Gamma$ and, for $\gamma, \delta, \Upsilon$ as above, we say $\gamma \sim \delta$ via $\Upsilon$.

For fixed $q$, the equivalence classes of $\sim$ are parameterized by maps

$$
\psi: \mathbb{Z}_{\geq 1} \rightarrow \mathbb{M P}
$$

written

$$
\psi(j)=\left(\psi(j)_{1}^{b(j)_{1}}, \psi(j)_{2}^{b(j)_{2}}, \ldots, \psi(j)_{m(j)}^{b(j)_{m(j)}}\right)
$$

such that:

(i) $\psi(j)$ is the empty multi-partition for all but finitely many $j \in \mathbb{Z}_{\geq 1}$;

(ii) $\sum_{j \in \mathbb{Z}_{\geq 1}} j|\psi(j)|=n$; and

(iii) $\sum_{r=1}^{m(j)} b(j)_{r} \leq \phi(j)$ for all $j \in \mathbb{Z}_{\geq 1}$, where $\phi$ is as in (2.1).

We write $\Psi$ for the set of all maps $\psi: \mathbb{Z}_{\geq 1} \rightarrow \mathbb{M P}$ satisfying conditions (i) and (ii) above. For $\psi \in \Psi$ written as in (3.2), we define

$$
A(\psi)=\left\{(j, r, s) \mid j \in \mathbb{Z}_{\geq 1}, r=1, \ldots, m(j), s=1, \ldots, b(j)_{r}\right\} .
$$

Provided that condition (iii) above holds for $\psi \in \Psi$, we can choose $\bar{a}(j)_{r}^{s} \in \mathbb{F}_{q^{j}}^{\sharp} / F$ for each $(j, r, s) \in A(\psi)$ such that the $\bar{a}(j)_{r}^{s}$, s are all distinct. Then we can define $\gamma \in \Gamma$ by

$$
\gamma(\bar{a})= \begin{cases}\psi(j)_{r} & \text { if } \bar{a}=\bar{a}(j)_{r}^{s} \text { for some }(j, r, s) \in A(\psi) \\ () & \text { otherwise. }\end{cases}
$$

All possible choices for the $\bar{a}(j)_{r}^{S}$ gives the $\sim$-equivalence class $\tilde{\psi}$ corresponding to $\psi$. If condition (iii) does not hold for $\psi$, then, by convention, $\tilde{\psi}$ is the empty set. With this convention, we can view the set $\Psi$ as parameterizing the equivalence classes of $\sim$, and this parametrization does not depend on $q$.

Next we count the number of elements in $\tilde{\psi}$ for $\psi \in \Psi$. If we write $\psi(j)$ as in (3.2), then, using the description of the equivalence class $\tilde{\psi}$ as given by (3.4), one 
can see that the desired number is

$$
|\tilde{\psi}|=\prod_{j \in \mathbb{Z}_{\geq 1}} \Delta(b(j), \phi(j)),
$$

where: $\Delta$ is defined in $(2.2) ; b(j)=\left(b(j)_{1}, \ldots, b(j)_{m(j)}\right) \in \mathbb{Z}_{\geq 1}^{m(j)}$ as in (3.2); and $\phi(j)=\left|\mathbb{F}_{q^{j}}^{\sharp} / F\right|$, see (2.1). Since each $\phi(j)$ is a polynomial in $q$ and $\Delta(b(j), \phi(j))$ is a polynomial in $\phi(j)$, we see that $|\tilde{\psi}|$ is a polynomial in $q$; we note, however, that in general the coefficients of this polynomial are not integers.

If $\gamma \sim \delta$ (via $\Upsilon$ ), then we can identify the bases $\mathbb{B}(\gamma)$ and $\mathbb{B}(\delta)$ of $K^{n}$ used to define $x(\gamma)$ and $x(\delta)$, i.e., for $\bar{a} \in K^{\times} / F$, we identify $\mathbb{B}(\gamma)_{\bar{a}}$ with $\mathbb{B}(\delta)_{\bar{b}}$, where $\bar{b}=$ $\Upsilon(\bar{a})$. Therefore, for $\psi \in \Psi$, we can define $\mathbb{B}(\psi)=\mathbb{B}(\gamma)$ for some $\gamma \in \tilde{\psi}$. Suppose that $\gamma, \delta \in \tilde{\psi}$, then having identified $\mathbb{B}(\gamma)=\mathbb{B}(\delta)=\mathbb{B}(\psi)$, we have $H(\gamma)=H(\delta)$. Writing $H(\psi)=H(\gamma)$, from (3.1) and the description of $\gamma \in \tilde{\psi}$ as in (3.4) we see that

$$
H(\psi) \cong \prod_{(j, r, s) \in A(\psi)} \operatorname{GL}_{\left|\psi(j)_{r}\right|}\left(q^{j}\right) .
$$

We also have $u(\gamma)=u(\delta)$, so we can define $u(\psi)=u(\gamma)$. The conjugacy class of $u(\psi)$ in $H(\psi)$ is parameterized by the partitions in the $\psi(j)$, i.e., the conjugacy class of a unipotent element $u \in H(\psi)$ is given by the class of the projection of $u$ into each factor $\mathrm{GL}_{\left|\psi(j)_{r}\right|}\left(q^{j}\right)$, this is given by a partition of $\left|\psi(j)_{r}\right|$; for $u=u(\psi)$, this is precisely the partition $\psi(j)_{r}$.

For each value of $q$ such that $\tilde{\psi}$ is nonempty, we choose some $\gamma=\gamma(q) \in \tilde{\psi}$. Then we set $x(\psi)=x(\gamma)$ and allow this to vary as $q$ does; we note that $x(\psi)$ depends on the choice of $\gamma$. We write the Jordan decomposition of $x(\psi)$ as $x(\psi)=s(\psi) u(\psi)$. The semisimple part $s(\psi)$ depends on the choice of $\gamma$, but $H(\psi)=C_{G}(s(\psi))$ does not; $H(\psi)$ is given as in (3.6) for all values of $q$. The parameterization of the conjugacy class of $u(\psi) \in H(\psi)$ does not change as $q$ varies. The discussion in this paragraph gives a convention to vary $q$, which we use in the next section.

\section{Proof of Theorem 1.1}

For this section, we fix an $n$-dimension vector $d$ and let $P=\mathrm{P}_{n, d}(q)$ be the corresponding parabolic subgroup of $G=\mathrm{GL}_{n}(q)$ as defined in the introduction. Let $\psi \in \Psi$, and assume that $q$ is large enough so that $\tilde{\psi}$ is nonempty. Let $x=x(\psi)$, $s=s(\psi), u=u(\psi), \mathbb{B}=\mathbb{B}(\psi)$, and $H=H(\psi)=C_{G}(s)$ be defined by choosing $\gamma \in \tilde{\psi}$ as at the end of Sect. 3 .

The basis $\mathbb{B}=\mathbb{B}(\psi)$ of $K^{n}$ determines an $F$-stable maximal torus $\mathbf{T}=\mathbf{T}(\psi)$ of $\mathbf{G}=\mathrm{GL}_{n}(K)$ consisting of the elements of $\mathbf{G}$ which act diagonally on $K^{n}$ with respect to $\mathbb{B}$; we write $T=\mathbf{T}^{F}$. We note that $\mathbf{T}$ is not split unless $\psi(j)=($ ) for all $j \geq 2$, but $\mathbf{T}$ is a maximally split maximal torus of $\mathbf{H}=C_{\mathbf{G}}(s(\psi))$.

Suppose that $x \in{ }^{y} P$ for some $y \in G$. The uniqueness of Jordan decompositions implies that $s \in{ }^{y} P$, which in turn implies that ${ }^{y} P \cap H$ is a parabolic subgroup of $H$. It follows that there exists $z \in H$ such that $T \subseteq{ }^{z y} P$. 
As $s$ is central in $\mathbf{H}$ and the centre of $\mathbf{H}$ is connected, we have that $s$ is in any parabolic subgroup of $\mathbf{H}$. In particular, this implies that $s \in Q$ for any parabolic subgroup $Q$ of $H$, and so $x \in Q$ if and only if $u \in Q$.

We let $\mathcal{Q}$ be a set of representatives of the $H$-orbits in $\left\{{ }^{g} P \mid g \in G\right\}$ that are of the form $H \cdot\left({ }^{g} P\right)$ for some ${ }^{g} P$ with $T \subseteq{ }^{g} P$; we assume that $T \subseteq P^{\prime}$ for all $P^{\prime} \in \mathcal{Q}$. From the discussion in the previous two paragraphs we see that

$$
f_{P}^{G}(x)=\sum_{P^{\prime} \in \mathcal{Q}} f_{P^{\prime} \cap H}^{H}(u),
$$

where the function $f_{P}^{G}$ is defined as in the introduction. We note that this equation does not depend on the choice of $\gamma \in \tilde{\psi}$ used to define $x=x(\gamma)$.

Below we give a parameterization of the set $\mathcal{Q}$. This is first done in terms of the chosen $\gamma \in \tilde{\psi}$, and then we explain how the parameterization can be described in terms of $\psi$. The idea is that as any $P^{\prime} \in \mathcal{Q}$ contains $T$, therefore, the corresponding parabolic subgroup $\mathbf{P}^{\prime}$ of $\mathbf{G}$ (containing $\mathbf{T}$ and so that $P^{\prime}=\left(\mathbf{P}^{\prime}\right)^{F}$ ) is the stabilizer in G of some flag $\{0\} \subseteq V_{1} \subseteq \cdots \subseteq V_{t}=K^{n}$ with respect to the basis $\mathbb{B}=\mathbb{B}(\gamma)$, i.e., each $V_{i}$ has a basis which is a subset of $\mathbb{B}$. In order for $\mathbf{P}^{\prime}$ to be $F$-stable, we require that whenever some $v \in \mathbb{B}$ is in $V_{i}$, then so is $F(v)$. Further, the action of $H$ allows the basis elements in $\mathbb{B}_{a}$ for fixed $a \in K^{\times}$to be permuted.

We let $\mathcal{C}=\mathcal{C}(\gamma)$ be the set of all maps

$$
c: K^{\times} / F \times\{1, \ldots, t\} \rightarrow \mathbb{Z}_{\geq 0}
$$

such that: $\sum_{\bar{a} \in K^{\times} / F} \operatorname{deg}(a) c(\bar{a}, i)=d_{i}$ for each $i=1, \ldots, t$; and $c(\bar{a}, i) \leq c(\bar{a}, i+1)$ and $c(\bar{a}, t)=|\gamma(\bar{a})|$ for all $\bar{a} \in K^{\times} / F$. Given $c \in \mathcal{C}, a \in K^{\times}$, and $i \in\{1, \ldots, t\}$, we define $\mathbb{B}_{a, i}$ to consist of the first $c(\bar{a}, i)$ elements of $\mathbb{B}_{a}$. We define $V_{i}$ to have basis $\mathbb{B}_{i}=\bigcup_{a \in K^{\times}} \mathbb{B}_{a, i}$. The parabolic subgroup $Q(c)$ of $G$ is defined to be the stabilizer in $G$ of the flag $\{0\} \subseteq V_{1} \subseteq \cdots \subseteq V_{t}=K^{n}$. We can take $\mathcal{Q}=\{Q(c) \mid c \in \mathcal{C}\}$ to be our set of representatives.

We write $\psi(j)$ as in (3.2) and define $A(\psi)$ as in (3.3). Then $\mathcal{E}=\mathcal{E}(\psi)$ is defined to be the set of all maps

$$
e: A(\psi) \times\{1, \ldots, t\} \rightarrow \mathbb{Z}_{\geq 0}
$$

such that: $\sum_{(j, r, s) \in A(\psi)} j e(j, r, s, i)=d_{i}$ for all $i=1, \ldots, t ; e(j, r, s, i) \leq e(j, r, s$, $i+1)$ and $e(j, r, s, t)=\left|\psi(j)_{r}\right|$ for all $(j, r, s) \in A(\psi)$. We are assuming that $\tilde{\psi}$ is nonempty, so we may fix a choice of distinct $\bar{a}(j)_{r}^{s} \in \mathbb{F}_{q^{j}}^{\sharp} / F$ and define $\gamma$ from $\psi$ as in (3.4). For each $e \in \mathcal{E}$, we define $c=C(e) \in \mathcal{C}=\mathcal{C}(\gamma)$ by

$$
c(\bar{a}, i)= \begin{cases}e(j, r, s, i) & \text { if } \bar{a}=\bar{a}(j)_{r}^{s} \text { for some }(j, r, s) \in A(\psi) \\ 0 & \text { otherwise. }\end{cases}
$$

The map $C: \mathcal{E} \rightarrow \mathcal{C}$ is a bijection. For $e \in \mathcal{E}$, we set $Q(e)=Q(C(e))$ and note that this does not depend on the choice of $\gamma$, i.e., the choice of the $\bar{a}(j)_{r}^{s}$. It follows that the set $\mathcal{E}$ gives a parameterization of the set $\mathcal{Q}$. 
Now by (4.1) we get

$$
f_{P}^{G}(x(\psi))=\sum_{e \in \mathcal{E}} f_{Q(e) \cap H}^{H}(u(\psi)) .
$$

For values of $q$ such that $\tilde{\psi}$ is nonempty, each $f_{Q(e) \cap H}^{H}(u(\psi))$ is a polynomial in $q$ (with integer coefficients) by [5, Theorem 3.10]. Here we use the convention to vary $q$ as discussed at the end of Sect. 3. As the set $\mathcal{E}$ does not depend on $q$, we deduce that $f_{P}^{G}(x(\psi))$ is a polynomial in $q$.

Now by (1.1) we have

$$
k(P, G)=\sum_{\gamma \in \Gamma} f_{P}^{G}(x(\gamma)),
$$

using the parameterization of the $G$-conjugacy classes given in Sect. 3. It is implicit in (4.3) that $f_{P}^{G}(x(\gamma))=f_{P}^{G}(x(\psi))$ for any $\gamma \in \tilde{\psi}$, so we have that

$$
k(P, G)=\sum_{\psi \in \Psi}|\tilde{\psi}| f_{P}^{G}(x(\psi)),
$$

where by convention we set $f_{P}^{G}(x(\psi))=0$ if $\tilde{\psi}=\varnothing$. By (3.5) we have that $|\tilde{\psi}|$ is a polynomial in $q$ and we have shown above that $f_{P}^{G}(x(\psi))$ is a polynomial in $q$. Hence, $k(P, G)$ is a polynomial in $q$.

To complete the proof of Theorem 1.1, we need to show that the coefficients of the polynomial $k(P, G)$ are integers. We fix a prime $p$ and, in this paragraph, just consider values of $q$ that are powers of $p$; for the proof that the coefficients of the polynomials $k(P, G)$ are integers, it suffices to just consider such $q$. Arguing as in the introduction of [4], we can find a family of varieties $V_{1}, \ldots, V_{m}$ defined over $\mathbb{F}_{p}$ such that the $P$-conjugacy classes in $G$ correspond to the $\mathbb{F}_{q}$-rational points of the $V_{i}$. More precisely, using Rosenlicht's theorem (see [13]), we can find a $\mathbf{P}$-stable open subvariety $U_{1}$ of $\mathbf{G}$ defined over $\mathbb{F}_{p}$ and an orbit space $V_{1}$ for the action of $\mathbf{P}$ on $U_{1}$. This means that the points of $V_{1}$ (over $K$ ) correspond to the $\mathbf{P}$-conjugacy classes in $U_{1}$. Now using the fact that $C_{\mathbf{P}}(x)$ is connected for any $x \in \mathbf{G}$, we see that the $\mathbb{F}_{q}$-rational points of $V_{1}$ correspond to the conjugacy classes of $P$ in the set of $\mathbb{F}_{q}$-rational points of $U_{1}$; this follows from [2, Proposition 3.21]. Now we can apply Rosenlicht's theorem to the action of $\mathbf{P}$ on $\mathbf{G} \backslash U_{1}$ to find $U_{2}$ and $V_{2}$ in analogy to $U_{1}$ and $V_{1}$. Continuing in this way, we obtain the varieties $V_{1}, \ldots, V_{m}$ whose $\mathbb{F}_{q}$-rational points correspond to the $P$-conjugacy classes in $G$. Given this parameterization of the $P$-conjugacy classes in $G$, one can apply some standard arguments, using the Grothendieck trace formula (see [2, Theorem 10.4]), to prove that the coefficients of the polynomial $k(P, G)$ are integers, see for example [11, Proposition 6.1].

We note that the polynomial summands $|\tilde{\psi}| f_{P}^{G}(x(\psi))$ in the expression for $k(P, G)$ given in (4.4) do not have integer coefficients in general; this can already be seen for $G=\mathrm{GL}_{2}(q)$ in the examples below.

We conclude our discussion with some examples which demonstrate that it is possible to explicitly calculate the polynomials $k(P, G)$. We observe that, in the examples below, $k(P, G)$ is divisible by $q-1$. One can see that this has to be the case by checking that $q-1$ divides the polynomial $|\tilde{\psi}|$ for all $\psi$. 
Table 1 The case $\mathrm{GL}_{2}(q)$

\begin{tabular}{lllll}
\hline$\psi(1)$ & $\psi(2)$ & $x(\psi)$ & $|\tilde{\psi}|$ & $f_{B}^{G}(x(\psi))$ \\
\hline$\left(\left(1^{2}\right)\right)$ & () & $\left(\begin{array}{ll}a & 0 \\
0 & a\end{array}\right), a \in \mathbb{F}_{q}^{\times}$ & $q-1$ & $q+1$ \\
$((2))$ & () & $\left(\begin{array}{ll}a & 1 \\
0 & a\end{array}\right), a \in \mathbb{F}_{q}^{\times}$ & $q-1$ & 1 \\
$\left((1)^{2}\right)$ & () & $\left(\begin{array}{cc}a & 0 \\
0 & b\end{array}\right), a \neq b \in \mathbb{F}_{q}^{\times}$ & $\frac{(q-1)(q-2)}{2}$ & 2 \\
() & $((1))$ & $\left(\begin{array}{cc}a & 0 \\
0 & a^{q}\end{array}\right), a \in \mathbb{F}_{q^{2}}^{\sharp}$ & $\frac{q^{2}-q}{2}$ & 0 \\
\hline
\end{tabular}

\section{Example 4.1}

(i) We begin by explicitly calculating $k(B, G)$ and $k(G)=k(G, G)$ for $G=$ $\mathrm{GL}_{2}(q)$. The possible values of $\psi$ and all the information needed to calculate $k(B, G)$ and $k(G)$ is given in Table 1 . It is straightforward to calculate all of the information in this table by hand.

Now, using (4.4), we can calculate:

$$
k(B, G)=(q-1)(q+1)+(q-1) 1+\frac{(q-1)(q-2)}{2} 2=2 q(q-1) .
$$

Of course, we have $f_{G}^{G}(x(\psi))=1$ for all $\psi$, so we obtain:

$$
k(G)=(q-1)+(q-1)+\frac{(q-1)(q-2)}{2}+\frac{q^{2}-q}{2}=(q-1)(q+1) .
$$

(ii) For $n \geq 3$ (not too large), it is straightforward to calculate $k(B, G)$, using the values of the functions $f_{B}^{G}(u)$ for unipotent $u$. It is possible to obtain these values, using the chevie package in GAP3 [3] along with some code provided by M. Geck and the formula for $f_{B}^{G}(u)$ given in [5, Lemma 3.2]. The size of $\Psi$ gets large quickly as $n$ increases, so we have only calculated the values of $k(B, G)$ for $n \leq 4$. We do not include the details of these calculations here, since this would take a lot of space. For $n=3$, we get

$$
k(B, G)=(q-1)\left(q^{3}+6 q^{2}-q-3\right)
$$

and, for $n=4$, we obtain

$$
k(B, G)=(q-1)\left(q^{6}+3 q^{5}+9 q^{4}+19 q^{3}-9 q^{2}-18 q+5\right) .
$$

(iii) We finish by giving an example of how to calculate a particular value of $f_{P}^{G}(x(\psi))$. We consider the case $G=\mathrm{GL}_{9}(q), P=\mathrm{P}_{9, d}(q)$, where $d$ is the 9dimension vector $(4,7,9)$, and $\psi$ is given by

$$
\psi(1)=((2)), \quad \psi(2)=\left(\left(1^{2}\right)\right), \quad \psi(3)=((1)) ; \quad \psi(j)=() \quad \text { for } j \geq 4 .
$$

We write $x=x(\psi)$ with Jordan decomposition $x=s u$ and we write $H=C_{G}(s)$. We have the direct product decomposition $H=\mathrm{GL}_{2}(q) \times \mathrm{GL}_{2}\left(q^{2}\right) \times \operatorname{GL}_{1}\left(q^{3}\right)=$ 
$H_{1} \times H_{2} \times H_{3}$, say. We write $x_{i}$ for the projection of $x$ into $H_{i}$ for each $i$. We note that $x_{1}$ is a product of a central element and a regular unipotent element in $H_{1}$, $x_{2}$ is central in $H_{2}$, and $x_{3}$ is central in $H_{3}$. Given a parabolic subgroup $Q$ of $H$ containing $s$, we write $Q_{i}=Q \cap H_{i}$ for each $i$ and note that

$$
f_{Q}^{H}(x)=f_{Q_{1}}^{H_{1}}\left(x_{1}\right) f_{Q_{2}}^{H_{2}}\left(x_{2}\right) f_{Q_{3}}^{H_{3}}\left(x_{3}\right) .
$$

Using (3.5), we can calculate

$$
|\tilde{\psi}|=(q-1) \frac{q^{2}-q}{2} \frac{q^{3}-q}{3} .
$$

We have $A(\psi)=\{(1,1,1),(2,1,1),(3,1,1)\}$. There are three elements $e \in \mathcal{E}(\psi)$ that are shown in the following three matrices: the value of $e(j, 1,1, i)$ being given by the entry in the $j$ th row and $i$ th column:

$$
\left(\begin{array}{lll}
1 & 2 & 2 \\
0 & 1 & 2 \\
1 & 1 & 1
\end{array}\right), \quad\left(\begin{array}{lll}
0 & 0 & 2 \\
2 & 2 & 2 \\
0 & 1 & 1
\end{array}\right), \quad\left(\begin{array}{lll}
2 & 2 & 2 \\
1 & 1 & 2 \\
0 & 1 & 1
\end{array}\right) .
$$

Next we use (4.5) to work out the value of $f_{Q(e)}^{H}(x(\psi))$ for each of the three possible values of $e$. In the first case, we have that $Q_{1}$ is a Borel subgroup of $H_{1}$, so that $f_{Q_{1}}^{H_{1}}\left(x_{1}\right)=1 ; Q_{2}$ is a Borel subgroup of $H_{2}$, so that $f_{Q_{2}}^{H_{2}}\left(x_{2}\right)=q^{2}+1$; and $Q_{3}$ is (necessarily) all of $H_{3}$, so we get $f_{Q_{3}}^{H_{3}}\left(x_{3}\right)=1$. We can work out the value of $f_{Q(e)}^{H}(x)$ for the other two possible values of $e$ similarly and then we can use (4.3) to calculate

$$
f_{P}^{G}(x)=\left(q^{2}+1\right)+1+\left(q^{2}+1\right)=2 q^{2}+3 .
$$

Acknowledgements This research was funded in part by EPSRC grant EP/D502381/1. The first author would like to thank New College, Oxford for financial support whilst part of this research was carried out. We thank the referees for useful comments and suggestions.

\section{References}

1. Alperin, J. L. (2006). Unipotent conjugacy in general linear groups. Communications in Algebra, 34(3), 889-891.

2. Digne, F., \& Michel, J. (1991). Representations of finite groups of Lie type. In London mathematical society student texts (Vol. 21). Cambridge: Cambridge University Press.

3. The GAP group. (1997). GAP_groups, algorithms, and programming-version 3, release 4, patchlevel 4. Lehrstuhl D für Mathematik, Rheinisch Westfälische Technische Hochschule, Aachen.

4. Goodwin, S. M. (2007). Counting conjugacy classes in Sylow p-subgroups of Chevalley groups. Journal of Pure and Applied Algebra, 210(1), 201-218.

5. Goodwin, S. M., \& Röhrle, G. (2007, to appear). Rational points on generalized flag varieties and unipotent conjugacy in finite groups of Lie type. Transactions of the American Mathematical Society.

6. Green, J. A. (1955). The characters of the finite general linear groups. Transactions of the American Mathematical Society, 80, 402-447.

7. Higman, G. (1960). Enumerating p-groups, I: inequalities. Proceedings of the London Mathematical Society, 10(3), 24-30.

8. Higman, G. (1960). Enumerating p-groups, II: problems whose solution is PORC. Proceedings of the London Mathematical Society, 10(3), 566-582. 
9. Kac, V. G. (1983). Root systems, representations of quivers and invariant theory. In Lecture notes in mathematics: Vol. 996. Invariant theory (pp. 74-108). Montecatini, 1982. Berlin: Springer.

10. Macdonald, I. G. (1995). Symmetric functions and Hall polynomials (2nd ed.). New York: Oxford University Press.

11. Reineke, M. (2006). Counting rational points of quiver moduli. International Mathematics Research Notices. Art. ID 70456.

12. Robinson, G. R. (1998). Counting conjugacy classes of unitriangular groups associated to finitedimensional algebras. Journal of Group Theory, 1(3), 271-274.

13. Rosenlicht, M. (1963). A remark on quotient spaces. Anais da Academia Brasileira de Ciências, 35, 487-489.

14. Thompson, J. $k\left(\mathrm{U}_{n}\left(F_{q}\right)\right)$. Preprint. http://www.math.ufl.edu/fac/thompson.html.

15. Vera-López, A., \& Arregi, J. M. (2003). Conjugacy classes in unitriangular matrices. Linear Algebra and its Applications, 370, 85-124. 